\title{
Mutual Interference Between Surface and Satellite Communication Systems
}

\author{
William J. Hartman and Martin T. Decker
}

(Received April 27, 1961)

\begin{abstract}
Contribution from Central Radio Propagation Laboratory, National Bureau of Standards, Boulder, Colo.
Estimates of the mutual interference expected to occur between the ground terminals of space communications systems and surface point-to-point systems are presented in a fashion suitable for engineering applications. These estimates are obtained from recently developed methods for predicting the transmission loss over tropospheric paths in terms of parameters such as geographic separation, elevation angle of the antenna, antenna patterns and frequency. It is concluded that these systems can share the same frequency assignment under suitable conditions.
\end{abstract}

\section{Introduction}

Many of the problems of mutual interference between proposed satellite communications systems and surface communications systems can be investigated in terms of presently available theories. The prediction method used here is that developed by Rice, Longley, and Norton [1959], for tropospheric scatter propagation. The method is designed to predict the median value of hourly median basic transmission loss, $L_{b}$, and to give a distribution of the medians about this value; the median basic transmission loss is defined in terms of the ratio of the power, $p_{r}$, radiated from the transmitting antenna to the available power, $p_{a}$, at the receiving antenna, when isotropic antennas are used at both ends of the path. The method is applicable for any path configuration. The median transmission loss, $L$, is obtained from this by subtracting the path antenna gain, $G_{p}$, [Hartman and Wilkerson, 1959] from the basic transmission loss. It should be noted that these methods give excellent agreement with data measured over a frequency range from 100 $\mathrm{Mc} / \mathrm{s}$ to $10,000 \mathrm{Mc} / \mathrm{s}$. Most of these data were taken over conventional scatter paths with the antennas directed at the horizon in the great circle plane, although some preliminary data obtained using elevated beams are shown later in the paper.

Tolerable interference will depend not only on the relative levels of the desired and undesired signals, but also on the type of modulation used for both of these signals and in most cases must be determined experimentally, in some cases subjectively (for example, subjective rating of television pictures). Further, neither desired nor interfering signals will be steady signals, but will be represented by a distribution of values. Because of this, satisfactory operation should usually be specified in terms of an hourly-median-desired-signal to an hourly-median-undesired-signal ratio required to

1 This is a résumé of work sponsored by the Joint Technical Advisory Committee of the Electronic Industries Association and the Institute of Radio Engineers. provide a given grade of service or better for some percentage of the hours. A discussion of this problem is given by Norton [1959]; and Miller, Ports, and Savage [1960]. However, in view of the many unknowns the actual problem has been somewhat simplified by relating the hourly-median-interferingsignal power to the noise power of the receiver under consideration. Thus the question answered here is what separation distances and antenna elevation angles are required so that the interfering hourlymedian-signal power is equal to or less than the receiver noise power for a given percent of the hours.

\section{Path Parameters}

The following values of the path parameters are used here to illustrate the methods of a more general treatment [JTAC, 1961].

It is assumed that the satellite ground terminal has an antenna with the general characteristics of a $60-\mathrm{ft}$ parabolic dish, and that the surface system antennas are at most $10-\mathrm{ft}$ dishes. The satellite terminal antenna beam will be directed above the horizon at an angle $\psi$ as shown in figure 1 , and the

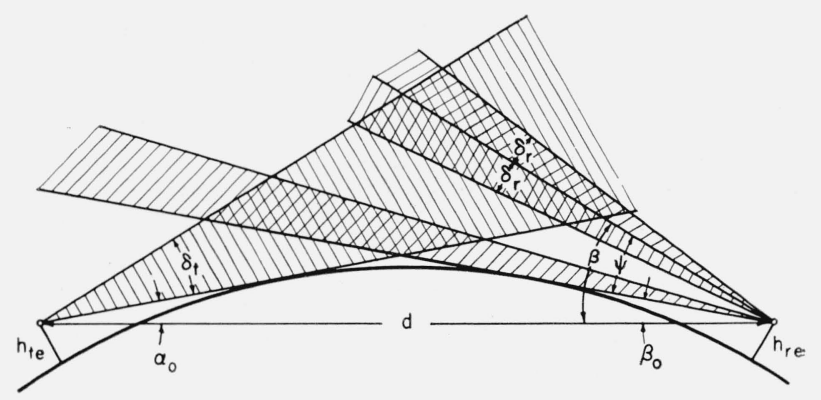

Figure 1. Geometry. 
surface system antenna will be directed at the horizon. Both antennas are to be located $30 \mathrm{ft}$ above a smooth spherical earth. Because antenna heights, terrain effects, and diffraction become increasingly important for shorter path lengths, a distance of 100 miles is chosen as a minimum for which calculations of this type will give representative values of transmission loss. At any distance a careful selection of the terminal site is necessary, and in some cases it will be possible to use terrain to give protection at distances shorter than those shown in this paper. If the specific path parameters are known, the transmission loss can be predicted accurately for any distance using the methods of the references given above [Hartman and Wilkerson, 1959; Rice, Longley, and Norton, 1959]. Both antenna axes are centered in the great circle plane. Although this situation usually results in the maximum interference, it may be impossible to avoid it if the satellite systems share frequencies with existing systems.
Figure 2 shows values of hourly median transmission loss which will be exceeded by 99.9 percent of the hourly medians for a $10-\mathrm{ft}$ dish at one end, and the main beam, a side lobe with the same half-power beamwidth as the main beam and $20 \mathrm{db}$ down from the main beam, and an isotropic portion of the antenna pattern for a $60-\mathrm{ft}$ dish at the other end. These are plotted versus the elevation angle, $\psi$, for the main beam of the $60-\mathrm{ft}$ dish. Examples of this type of calculation for median values are shown in figure 3. Also shown in this figure are the results of some preliminary measurements. These data points represent 5-minute medians measured over a 165mile test path from Boulder to Haswell, Colo. The pertinent parameters for this path are as follows: Frequency, $409.9 \mathrm{Mc} / \mathrm{s}$, the angular distances with both antennas directed at their respective horizons is 33 milliradians, the fixed transmitting antenna is a 14 -ft parabolic dish, and the receiving antenna being elevated is a $60-\mathrm{ft}$ dish.

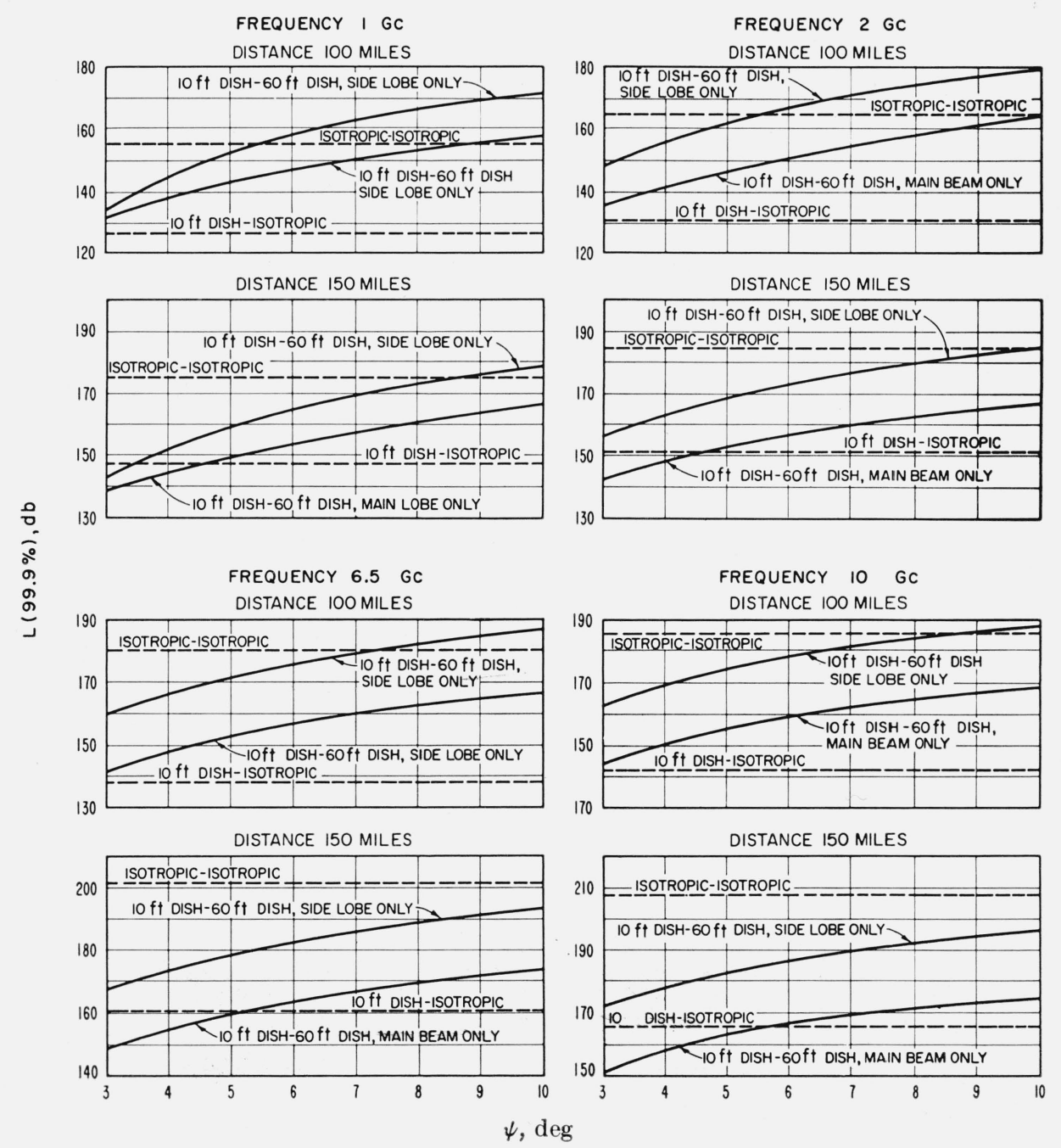

Figure 2. Transmission loss exceeded 99.9 percent of the time. 


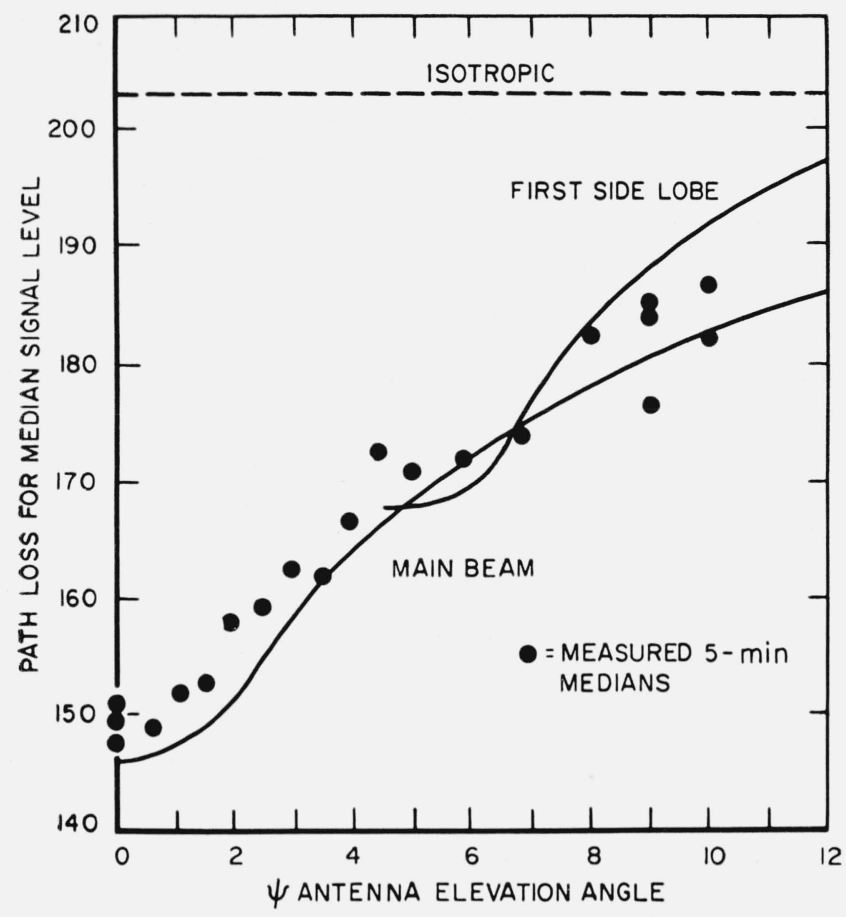

Figure 3. Boulder-Haswell Test Path, transmission loss exceeded 50 percent of the time.

The following procedure is used to determine the required separation distance for which the undesired signal is less than the receiver noise power at the receiver intermediate frequency for 99.9 percent of the hours.

Compute the value of transmission loss required to make the interfering signal equal to the noise power. This value is

$$
L_{\mathrm{req}}=P_{t}-L_{c}-M-P_{n}
$$

where $P_{t}$ is the power, in decibels, of the interfering transmitter, $L_{c}$ represents coupling losses in the system and may include transmission line losses, cross-polarization losses, etc., $M$ is a term to allow for the situation in which the transmitted energy is spread over a frequency band different from that which will be accepted by the receiver. It will depend on the type of modulation as well as the bandwidths, but an estimate is made here by letting $M=10 \log b_{t} / b_{r}$ where $b_{t}$ and $b_{r}$ are the transmitter and receiver bandwidths, respectively. $P_{n}$ is the available noise power in decibels at the receiver terminals and is given by

$$
P_{n}=N F+10 \log k T_{0} b=10 \log k T_{e} b
$$

where $N F$ is the effective noise figure [Norton, 1959; Hogg, 1960] of the receiving system, $k$ is Boltzmann's constant, $b$ is the noise bandwidth of the receiver in cycles per second, $T_{0}$ is a reference temperature, $288.48^{\circ} \mathrm{K}$, and $T_{e}$ is the effective noise temperature of the receiver and antenna combination. $T_{e}$ should not be confused with the effective antenna tempera- ture. This value of $L_{\text {req }}$ is then compared with the curves for the frequency under consideration to determine if $L_{\mathrm{req}} \leq L^{\prime}$ where $L^{\prime}$ is the smallest of the transmission loss values using the main beam or the side lobe or the isotropic part of the antenna pattern.

\section{Examples}

1. Consider first the case of interference from the earth terminal transmitter to a point-to-point relay receiver. The receiver noise power is determined as above assuming a bandwidth of $20 \mathrm{Mc} / \mathrm{s}$ and a noise figure of $10 \mathrm{db}$. Then

$$
P_{n}=N F+10 \log b-204=-121 \mathrm{dbw} .
$$

Assume a transmitter power of $1 \mathrm{kw}$, coupling losses of $4 \mathrm{db}$, including line and polarization losses, and a transmitted bandwidth of $20 \mathrm{Mc} / \mathrm{s}$. Then

$$
L_{\mathrm{req}}=P_{t}-L_{c}-M-P_{n}=147 \mathrm{db} .
$$

This transmission loss value is now used with figure 2 to determine whether the above condition, $L_{\mathrm{req}} \leq L^{\prime}$ can be met. It is clear that for a separation distance of 100 miles and for all four frequencies the transmission loss from the isotropic portion of the transmitting antenna is less than the $147 \mathrm{db}$ required loss. For a separation of 150 miles the energy transmitted by way of the main beam becomes significant and the required conditions can be met at all frequencies if the main beam of the $60-\mathrm{ft}$ antenna is elevated above the horizon by approximately $5^{\circ}$.

This analysis assumes that the antenna of the surface point-to-point system is directed toward the earth terminal. Rotating this antenna so that the main beam does not point directly toward the earth terminal would increase the transmission loss. When the pattern of the $10-\mathrm{ft}$ dish is at the isotropic level in the direction of the earth terminal the "isotropicisotropic" curves of figure 2 will apply rather than the "10-ft dish-isotropic" curves. The transmission loss via main and side lobes will of course increase by approximately the same amount. Therefore, the requirement could be met at 100 miles and at all frequencies by orienting the $10-\mathrm{ft}$ dish to reduce the interfering signal by $10 \mathrm{db}$ at $1 \mathrm{Gc}$ and by lesser amounts at the other frequencies.

Other earth terminal to satellite systems might require more or less transmitter power. Suggested systems [FCC hearing] range from approximately 3 to $36 \mathrm{dbw}$. The effect of changing this parameter in the above example would give a range of $L_{\text {req }}$ from 120 to $153 \mathrm{db}$ at the 99.9 percent level.

If instead of a $60-\mathrm{ft}$ dish, a $120-\mathrm{ft}$ dish or a $30-\mathrm{ft}$ dish were used for the transmitter, the only noticeable effect would be in the calculations for $L(p)$ for the main beam. In the former case $L(p)$ for the main beam would be decreased by less than $6 \mathrm{db}$ and in the latter case, $L(p)$ would be increased by approximately $6 \mathrm{db}$. This can alter the conclusions only by increasing (or decreasing) the required elevation angle of the transmitter by at most $2^{\circ}$. 
2. Consider next the case of interference from a point-to-point microwave relay transmitter to the earth-terminal receiver of a satellite system. It is assumed that a wide deviation FM system is used in the satellite to earth link, so that the earth terminal receiver has an $\mathrm{RF}$ bandwidth of $100 \mathrm{Mc} / \mathrm{s}$. With the use of a low noise antenna and maser amplifier, we assume that the effective noise temperature of the receiver is reduced to $30^{\circ} \mathrm{K}$.

$$
P_{n}=10 \log T_{e} b-228.6=-134 \mathrm{dbw} .
$$

Compute the transmission loss, $L_{\text {req }}$, assuming a transmitter power of $1 \mathrm{w}$, coupling losses of $4 \mathrm{db}$ and a transmitted bandwidth of $20 \mathrm{Mc} / \mathrm{s}$. Then

$$
L_{\mathrm{req}}=P_{t}-L_{c}-M-P_{n}=137 .
$$

This value of $L_{\text {req }}$ is compared with figure 2 to determine whether the requirement can be met. It is seen that under these assumptions the point-topoint antenna could not be directed toward the earth-terminal at a distance of 100 miles. As in the previous example the curves indicate that the requirements could be met by not allowing the $10-\mathrm{ft}$ dish to be directed toward the earth terminal.

The effects of varying the transmitter antenna size and the transmitted power were noted in example 1. Similar statements can be made about varying the receiver antenna size and transmitted power in this example. Values of the power for typical point-to-point microwave relays range from $-3 \mathrm{dbw}$ to $+7 \mathrm{dbw}$.

\section{Conclusions}

Our theoretical analysis indicates that space communications systems and surface systems of the conventional microwave relay type can share the same frequencies if care is used in locating the possible interfering sources. As seen in the examples, separation distances of from 100 miles to 150 miles usually suffice, and under ideal conditions, distances of less than 100 miles could give adequate protection. Estimates have been made for other systems such as high-powered radar and these indicate that harmful interference should not be experienced if the radar and earth terminals are separated by distances of 500 miles or more [JTAC, 1961].

The data shown in figure 3 represent samples during times when aircraft were not present in the propagation path, and although the data agree with the predicted values for the stated conditions, the estimates may not be accurate for some paths where aircraft are present.

The measurements reported here were made under the supervision of A. F. Barghausen.

\section{References}

Federal Communications Commission hearing, Allocation of frequencies in the bands above 890 Mc, F.C.C. Docket No. 11866 (undated).

Hartman, W. J., and R. E. Wilkerson, Path antenna gain in an exponential atmosphere, J. Research NBS 63D (Radio Prop.), No. 3, 273-286 (Nov.-Dec. 1959).

Hogg, D. C., Problems in low-noise reception of microwaves, Trans. IRE, Fifth National Symposium on Space Electronics and Telemetry, Part 8-2, pp 1-11 (Sept. 1960).

Joint Technical Advisory Committee of IRE-EIA, a report on frequency allocations for space communications, F.C.C. Docket No. 13522 (March, 1961).

Miller, C. R., D. C. Ports, and J. W. Savage, Receiver analysis for interference prediction purposes, IRE International Convention Record, Part 8 (1960).

Norton, Kenneth A., System loss in radio wave propagation, J. Research NBS 63D (Radio Prop.), No. 1, 53-73 (JulyAug. 1959).

Rice, P. L., A. G. Longley, and K. A. Norton, Prediction of the cumulative distribution with time of ground wave and tropospheric wave transmission loss, Part 1-The prediction formula, NBS Tech. Note 15 (1959).

(Paper 65D5-148) 